\title{
The Issue of FM to AM Conversion on the National Ignition Facility
}

\author{
J. E. Rothenberg \\ D. F. Browning \\ R. B. Wilcox
}

This paper was prepared for submittal to the Third Annual International Conference on Solid State Lasers for Application (SSLA)

to Inertial Confinement Fusion (ICF)

Monterey, California

June 7-12, 1998

August 13, 1998

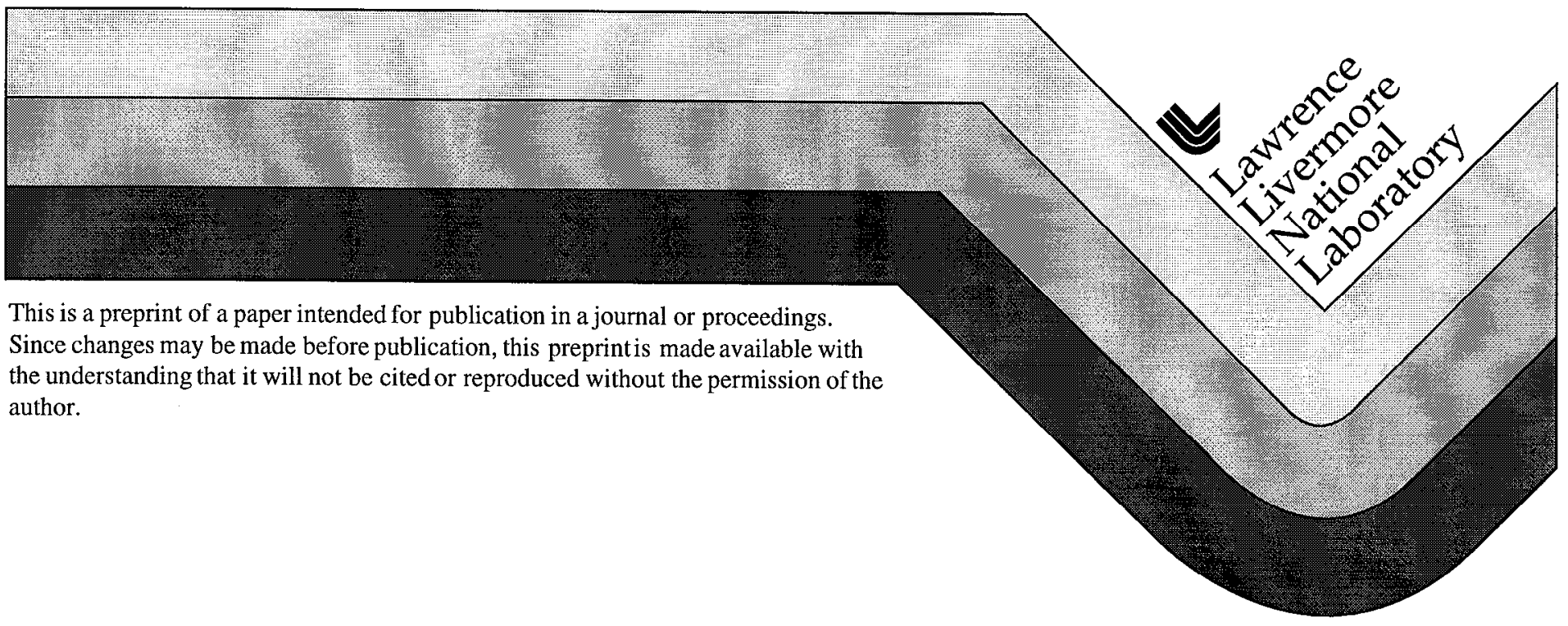




\section{DISCLAIMER}

This document was prepared as an account of work sponsored by an agency of the United States Government. Neither the United States Government nor the University of California nor any of their employees, makes any warranty, express or implied, or assumes any legal liability or responsibility for the accuracy, completeness, or usefulness of any information, apparatus, product, or process disclosed, or represents that its use would not infringe privately owned rights. Reference herein to any specific commercial product, process, or service by trade name, trademark, manufacturer, or otherwise, does not necessarily constitute or imply its endorsement, recommendation, or favoring by the United States Government or the University of California. The views and opinions of authors expressed herein do not necessarily state or reflect those of the United States Government or the University of California, and shall not be used for advertising or product endorsement purposes. 


\title{
The issue of FM to AM conversion on the National Ignition Facility
}

\author{
Joshua E. Rothenberg, Donald F. Browning, and Russell B. Wilcox \\ Lawrence Livermore National Laboratory, L-441,P. O. Box 808, Livermore, CA 94551 \\ Telephone: (510) 423-8613 , FAX: (510) 422-4982 , Email: JR1 @ LLNL.GOV
}

\begin{abstract}
The National Ignition Facility (NIF) baseline configuration for inertial confinement fusion requires phase modulation for two purposes. First, $\sim 1 \AA$ of frequency modulation (FM) bandwidth at low modulation frequency is required to suppress buildup of Stimulated Brioullin Scattering (SBS) in the large aperture laser optics. Also $\sim 3 \AA$ or more bandwidth at high modulation frequency is required for smoothing of the speckle pattern illuminating the target by the smoothing by spectral dispersion method (SSD). ${ }^{1}$ Ideally, imposition of bandwidth by pure phase modulation does not affect the beam intensity. However, as a result of a large number of effects, the FM converts to amplitude modulation (AM). In general this adversely affects the laser performance, e.g. by reducing the margin against damage to the optics. In particular, very large conversion of FM to AM has been observed in the NIF all-fiber master oscillator and distribution systems. The various mechanisms leading to AM are analyzed and approaches to minimizing their effects are discussed.
\end{abstract}

Keywords: Beam smoothing, smoothing by spectral dispersion, laser plasma instabilities, inertial confinement fusion.

\section{INTRODUCTION}

The common basis for the all effects which cause conversion of FM to AM is non-uniform spectral transmission -- either in phase or in amplitude. These effects include group velocity dispersion (nonuniform phase), variation of gain over the FM bandwidth, nonuniform spectral transmission from clipping by the far field pinholes (SSD disperses the bandwidth in the far field), etalon effects, spectral dependence of coatings, propagation away from the SSD grating and its image planes, beam motion on optics owing to SSD, frequency conversion, and polarization dispersion effects. For many of these effects, simple solutions (such as wedging optics to prevent etalon effects or use of a dispersive delay line to compensate for group velocity dispersion) can be implemented. However, it has recently been observed that interference effects in the NIF Master Oscillator Room (MOR) and fiber delivery system owing to small cumulative depolarization in the many fiber components can lead to a large amount of AM. The fiber which was planned to be used in the MOR and delivery system is highly birefringent (so called polarization maintaining or PM fiber) and thus the two allowed polarizations develop a propagation delay between them proportional to the birefringence (this is analogous to the round trip delay in an etalon). If these two polarizations are excited and subsequently mixed by imperfect polarization control, spectral nonuniformity owing to an etalon-like effect will result. Because of the coherent nature of the interference effect, even a 
"The issue of FM to AM conversion ...", J. E. Rothenberg et al

very small amount of depolarization (typically 100:1) can lead to very large $\mathrm{AM}$, which can ultimately decrease the safe maximum operating intensity of the laser amplifier.

A simple and fairly accurate rule for the magnitude of the AM effect resulting from spectral transmission variation is that the resulting peak-to-peak temporal AM is equal to the amount of intensity transmission variation over the FM spectrum. Thus, consider interference of the main beam with a small split-off power fraction $P$ which has been delayed by time $\Delta \tau$ (e.g. by either birefringence or a round trip in an etalon). The spectral transmission $S(\omega)$ results from coherent interference between the amplitudes of the main beam (assumed to be unity) and the small unwanted ghost beam of relative power $P$ or amplitude $\sqrt{P}$ :

$$
S(\omega)=|1+\sqrt{P} \exp [i \omega \Delta \tau)|^{2} .
$$

This transmission function then has a maximum (at $\omega \Delta \tau=0$ ) and a minimum (at $\omega \Delta \tau=\pi$ ) transmission of

$$
|1 \pm \sqrt{P}|^{2} \cong 1 \pm 2 \sqrt{P}
$$

and hence can lead to temporal AM with $4 \sqrt{P}$ peak-to-peak variation. In the approximate instantaneous frequency picture of $\mathrm{FM}$, the instantaneous frequency is varying sinusoidally over the bandwidth and the AM originates from the periodic variation in spectral transmission it experiences as it sweeps back and forth. For typical PM fiber components, which can have polarization intensity extinction $P \sim .01(-20 \mathrm{~dB})$, assuming complete mixing of this depolarized fraction with the main beam, the resulting AM could be as large as $4 \sqrt{.01}=40 \%$ peak-to-peak. Note that the above transmission function is periodic with free spectral range (FSR) equal to $v_{F S R}=\omega_{F S R} / 2 \pi=1 / \Delta \tau$. For the typical birefringent PM fiber we use, the delay between the slow and fast polarizations is $\sim 2.0 \mathrm{ps} / \mathrm{m}$ of fiber. Thus, 1 and $10 \mathrm{~m}$ birefringent fibers would have FSR's of $\sim 500 \mathrm{GHz}$ and $50 \mathrm{GHz}$, respectively. Depending on the FM bandwidth, the FSR, and the relative phase between the interfering terms at the center frequency of the FM, the AM can vary significantly. An example of the calculated AM is shown in Fig. 1 for a single $1 \mathrm{~m}$ fiber with an assumed mixing of polarizations $=1 / 250$. In this figure one sees that the AM varies by almost an order of magnitude simply as a result of a relative phase change between the interfering terms. Since many of the fiber sections will typically have lengths in the 1- $10 \mathrm{~m}$ range, the baseline SSD bandwidth of $\sim 3 \AA$ ( $81 \mathrm{GHz})$ will be a significant fraction of the FSR, and since the relative phases of the interference in the many fiber sections are randomly distributed, one statistically expects to observe a large fraction of the maximum AM predicted by Eq. (2).

The same type of analysis can be used to evaluate the effect of a conventional etalon in the optical path. In this case the power fraction of the double bounce ghost is $P=R^{2}$, where $R$ is the intensity reflectivity of each surface. Application of Eq. (2) shows that the peak-to-peak AM is $4 R$. Wedging the optic would eliminate this effect, or when impractical (such as for a birefringent material) very good AR coatings are required. It should be further noted the effect is increased for etalons located within a multipass amplifier. Since the loss at a given frequency is $\sim M$ times larger for $M$ passes though the same etalon one would expect that the resulting AM could be as large as $4 M R$ peak-to-peak. 
An alternative way of describing the effect of polarization dispersion in fibers is to note that PM fiber is, in effect, a very high order birefringent waveplate. For the typical PM fibers the birefringence leads to $\sim 600$ waves of retardance /meter of fiber at wavelength $1.05 \mu \mathrm{m}$. When such a waveplate is placed between polarizers one finds that the spectral transmission varies periodically with frequency period (FSR) equal to the center optical frequency $(\sim 300 \mathrm{THz})$ divided by the order of the waveplate, yielding $\sim 500 \mathrm{GHz}$ for a $1 \mathrm{~m}$ fiber, as was noted above. This effect can potentially also arise with a conventional multi-order waveplate (order $\sim 10$ ). However, the much larger FSR $(\sim 30 \mathrm{THz})$ in this case would result in a greatly reduced amount of AM.
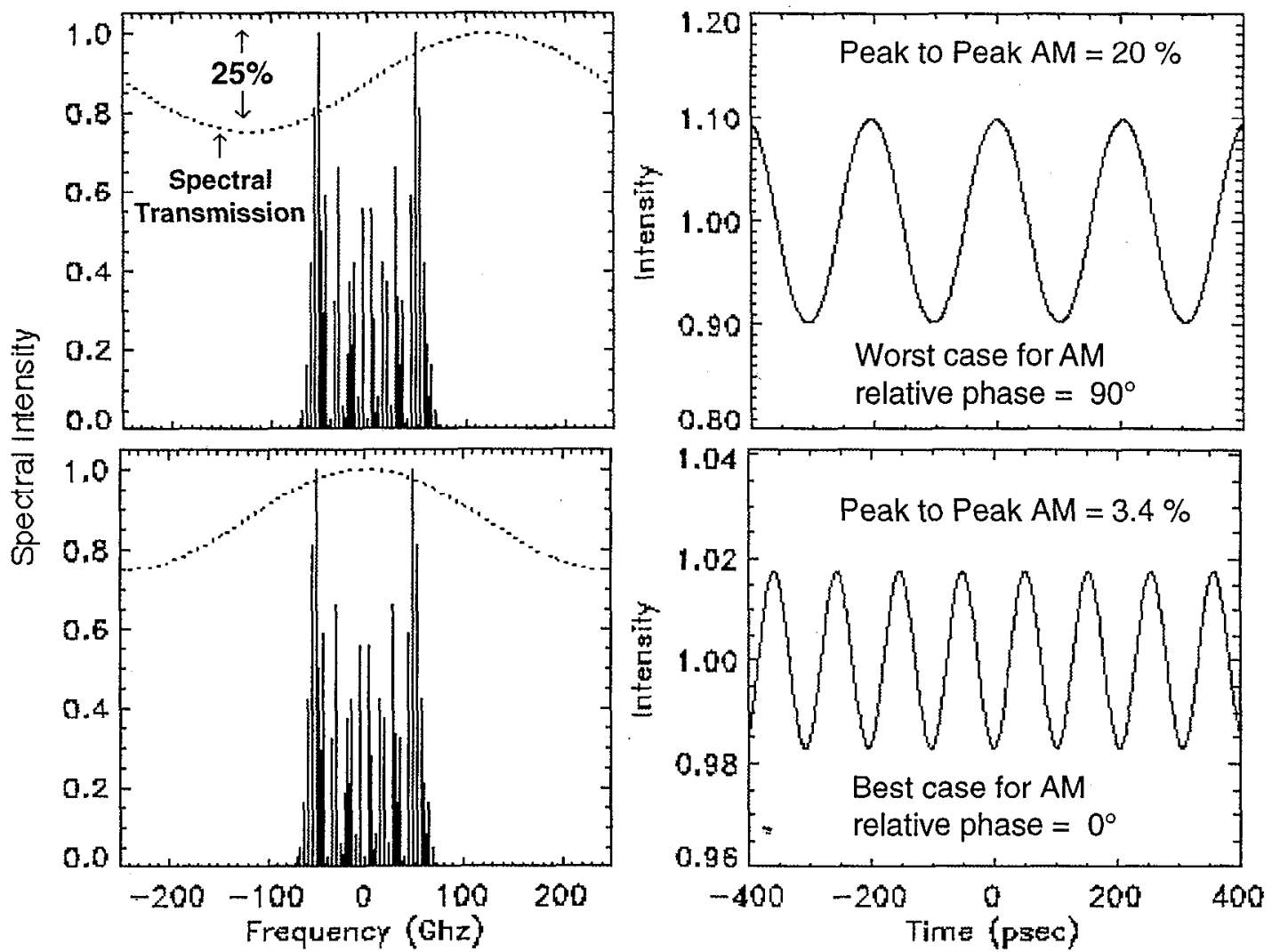

Fig. 1: Spectral and temporal modulation calculated for a $1 \mathrm{~m}$ length of birefringent fiber with depolarization of 250:1. The amount of AM is strongly dependent on the relative phase of the slow and fast waves at the center frequency.

\section{IMPACT OF AM ON LASER AND TARGET PERFORMANCE}

$\mathrm{AM}$ at $\mathrm{GHz}$ frequencies in itself is not currently considered to be a significant problem for target performance. However, the laser performance is potentially adversely affected by AM in regards to the issues of power balance among the beams and also margin against optical damage. Assume AM at $1 \omega$ of $\pm a / 2$ (Peak-to-Peak $A M=a$ ) thus

$$
\mathrm{I}_{1 \omega}=\mathrm{I}_{0} \cdot(1+(\mathrm{a} / 2) \cdot \sin \omega \mathrm{t}) \text {. }
$$

During the low intensity foot conversion to the third harmonic is approximately given by 
"The issue of FM to AM conversion ...", J. E. Rothenberg et al

$$
\mathrm{I}_{3 \omega} \sim \mathrm{I}_{1 \omega}{ }^{3}=\mathrm{I}_{0}{ }^{3} \cdot(1+(\mathrm{a} / 2) \cdot \sin \omega t)^{3}=\mathrm{I}_{0}^{3} \cdot\left(1+3 \mathrm{a}^{2} / 8+\ldots\right)
$$

Thus, e.g., $\pm 10 \%$ AM leads to an increase in time averaged conversion of $1.5 \%$. Since we expect the FM to vary from beam to beam, if the amount of AM is randomly distributed from 0 to $\pm \mathrm{a} / 2$, one finds that the resulting RMS variation in the $3 \omega$ power is given by

RMS of $3 \omega$ Power $=(a / 3)^{2}$.

This is not a very stringent requirement on the allowable amount of AM. E.g., $\pm 15 \%$ maximum $1 \omega$ AM results in RMS variation of the time averaged $3 \omega$ power of only $1 \%$.

AM can, however, present more of problem in regards to optical damage. If one integrates the laser intensity over a time longer than the modulation period, then the fluence is unchanged by AM. As a result one expects that the margin against damage driven simply by fluence will be unchanged. However, damage driven by filamentation and other fast nonlinear effects is sensitive to the (essentially) instantaneous intensity. Since AM as assumed in Eq. (3) causes the intensity to periodically exceed the average level by the factor $1+a / 2$, the threat of filamentation will be increased by this factor during the short interval of increased intensity. Thus, for example, if one requires the intensity-length product in the $3 \omega$ optics to be less than a given limit, one must reduce the average intensity by the factor $1+a / 2$ (perhaps modified by a correction to account for the change in the amount of AM in converting from $1 \omega$ to $3 \omega$ ) so as to keep the peak intensity-length product at or below its average level without AM present. Therefore, depending on whether simple fluence initiated damage or nonlinear effects limit the maximum laser intensity one sees that AM may have a direct impact on the maximum safe level of operation of the laser.

\section{FM TO AM CONVERSION IN FIBER AND FIBER COMPONENTS}

It is well known that FM will convert to AM owing to group velocity dispersion in fibers and other dispersive media. A simple calculation shows that the amount of $\mathrm{AM}$ is given by

$$
\text { Peak-to-Peak AM }=2.3 \times 10^{-8} \cdot \mathrm{D} \cdot \mathrm{z} \cdot \Delta \mathrm{v}_{\mathrm{bw}} \cdot v_{\mathrm{mod}}
$$

where $D$ is the dispersion in $p s / n m / k m, z$ is the propagation distance in $\mathrm{m}$, and $\Delta v_{b w}$ is the total FM bandwidth and $v_{\bmod }$ is the modulation frequency, both in GHz. Thus, for example propagation of $5 \AA$ of FM at modulation frequency $17 \mathrm{GHz}$ in $100 \mathrm{~m}$ of fiber with dispersion 40 $\mathrm{ps} / \mathrm{nm} / \mathrm{km}$ leads to Peak-to-Peak AM of $21 \%$. In the absence of nonlinear propagation in the fiber this effect can be eliminated by compensation with a dispersive delay line of negative group velocity dispersion, such as a diffraction grating pair.

As noted in Section 1, a more problematic source of AM in fibers is that of depolarization in PM fibers. The mechanism of this effect, as we have modeled it, is shown in Fig. 2. At the input of a single PM fiber one injects polarized light along the x-principle axis. There is a small amount of cross-coupling (of fractional power $P_{1}$ ) into the y-polarization mode, either because of imperfect alignment, or because of spurious mechanical stress at the fiber connector. The two modes propagate with differing velocities and thus develop a relative delay at the fiber output. Cross-coupling (of fraction $P_{2}$ ) again occurs at the output and the delayed pulse of 
"The issue of FM to AM conversion ...", J. E. Rothenberg et al

fractional power $P_{1} P_{2}$ is mixed with the main beam. The amplitude of the delayed pulse is $\sqrt{P_{1} P_{2}}$ and thus following the analysis of Eq. (1-2) one finds that the maximum peak to peak $\mathrm{AM}$ is given by

$$
\text { Peak-to-Peak } \mathrm{AM}=4 \sqrt{P_{1} P_{2}} \text {. }
$$

Of course, cross-coupling could also occur in a more distributed fashion along the entire length of the fiber, however, our measurements indicate that the dominant cross-coupling occurs at the fiber ends. The effect of more distributed depolarization would be to increase the FSR and to complicate the otherwise sinusoidal transmission function. In this case, one finds that the simplified model used here overestimates the amount of AM, and thus represents a conservative approach to estimating the expected AM.

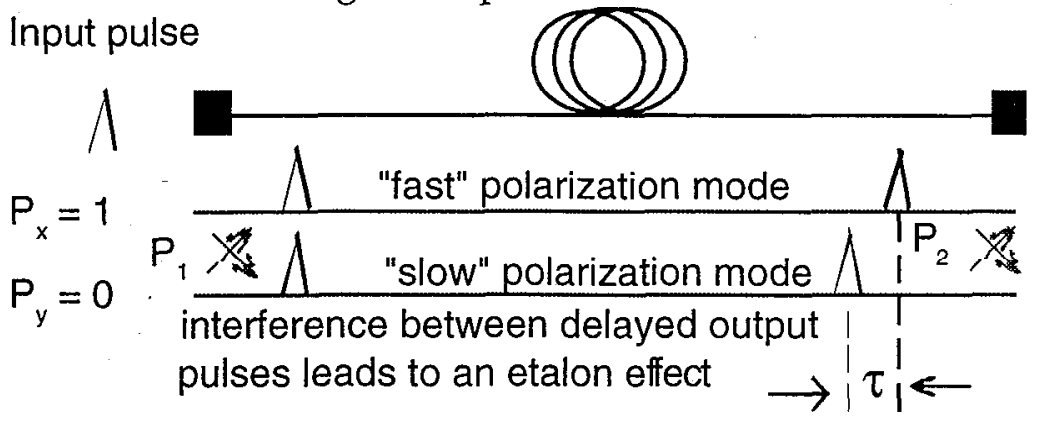

Figure 2: Schematic representation of the mixing process that leads to spectral modulation of the transmission through PM fiber.

The dependence of the amount of AM on the polarization extinction (assume $P_{1}=P_{2}$ ) and the fiber length (FSR) is calculated in Fig. 3. The bandwidth of the FM is assumed to be the NIF baseline value of $3 \AA(81 \mathrm{GHz})$. Many realizations, assuming randomly distributed relative phase of interference, are averaged to obtain the expected peak-to-peak AM. For fiber lengths less than $\sim 4 \mathrm{~m}(\mathrm{FSR}>125 \mathrm{GHz})$ one observes that the AM is reduced. This simply shows that the AM is reduced when the FSR is significantly larger than the bandwidth of interest. In addition, for long fibers the AM calculated is in agreement with the maximum found in Eq. (7).

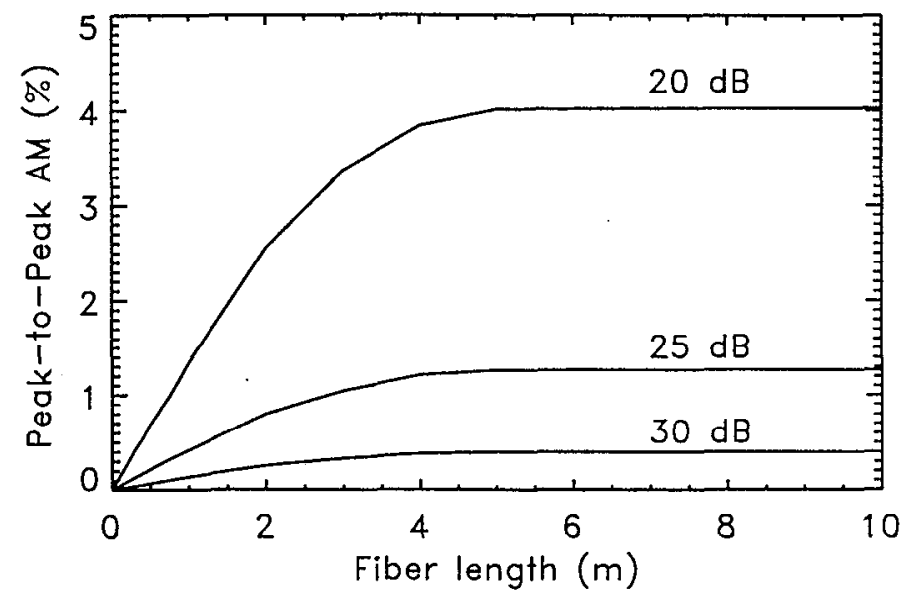

Figure 3: Peak to peak AM shown as a function of a single PM fiber length and polarization extinction (as indicated in $\mathrm{dB}$ ) for FM of bandwidth $81 \mathrm{GHz}$. Fiber is assumed to have polarization dispersion of $2 \mathrm{ps} / \mathrm{m}$. 
"The issue of FM to AM conversion ...", J. E. Rothenberg et al

From these calculations it would appear that use of a single PM fiber component with 20-30 dB extinction does not lead to very large levels of AM. However, the NIF fiber system involves 100 such components, and the cumulative depolarization which occurs can lead to very large levels of AM. In Fig. 4 the AM is calculated as a function of the number of PM fibers concatenated. One see that the AM grows linearly in proportion to the number of fibers concatenated. Thus, even for the best extinction available for PM fiber $(30 \mathrm{~dB})$, one finds that the cumulative effect of 100 components would lead to excessive amounts of AM. The accumulation of depolarization can be stemmed somewhat by applying a high extinction polarizing element periodically. In this case, one finds that the AM generated between polarizers adds in quadrature, and thus only grows in proportion to the square root of the number of components.

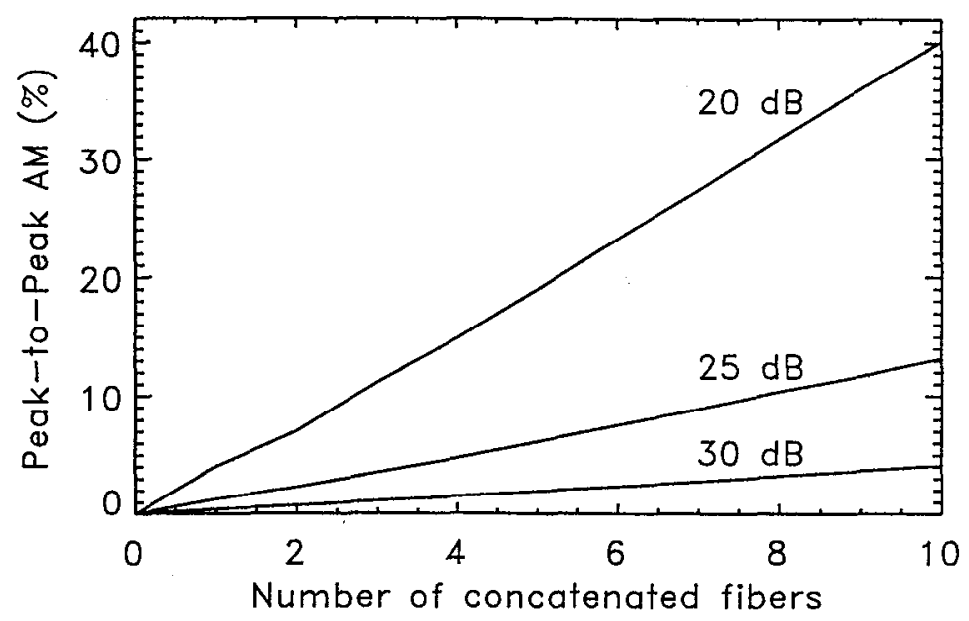

Figure 4: Peak to peak AM calculated as a function of the number of PM fibers concatenated and polarization extinction (as indicated in $\mathrm{dB}$ ) for FM of bandwidth $81 \mathrm{GHz}$. Fibers are assumed to be $10 \mathrm{~m}$ in length and have polarization dispersion of $2 \mathrm{ps} / \mathrm{m}$.

\section{MEASUREMENTS OF FM TO AM CONVERSION IN FIBER COMPONENTS}

Although direct measurement of temporal AM is possible with fast power detectors or streak cameras, it is quite difficult for small amounts of AM since these devices typically are noisy, and it has been also found to be difficult to eliminate spurious sources of AM (e.g. etalons). In addition if AM is observed with a temporal diagnostic, no signature of the cause of the AM can be obtained. In contrast, as noted above, the AM generated in PM fibers results from nonuniformity in spectral transmission, and thus spectral transmission may be a more informative measurement. In particular the source of the spectral modulation can be identified by its behavior (e.g. the FSR). In this method one must also take great care to eliminate spurious sources of spectral modulation. Figure 5 schematically shows the arrangement used to measure the spectral transmission of fiber components under test. The ratio of the incident and transmitted power as measured by slow detectors is recorded as a function of the slowly varied optical frequency as supplied by a temperature tuned single mode $\mathrm{CW}$ laser source. 
"The issue of FM to AM conversion ...", J. E. Rothenberg et al

Care has been take to eliminate unwanted etalon effects by use of wide angle diffusers at the input to the power detectors, and a large angle wedge is used to obtain the input reference power fraction. In addition a section of fiber is used as a spatial filter before the reference beam power splitter to eliminate the variation of input coupling with wavelength owing to the wavelength dependent mode size of the source laser.

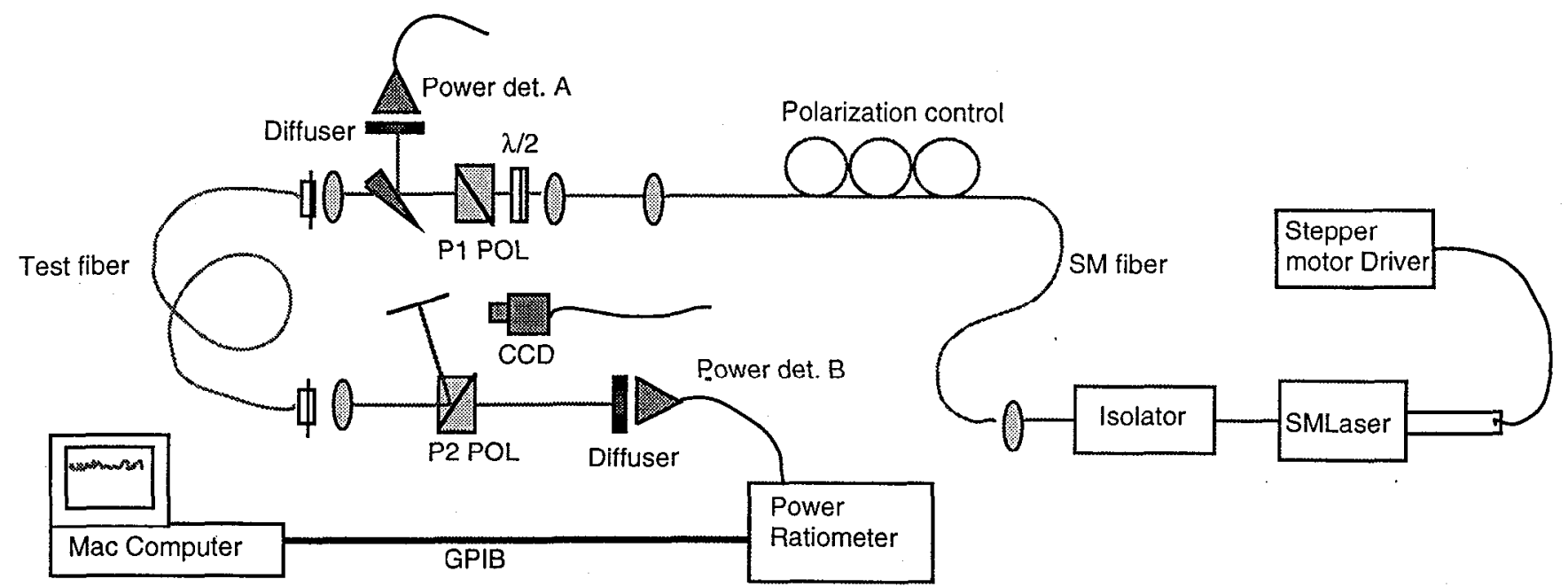

Figure 5: Experimental apparatus used to measure the spectral transmission of a test fiber component.

Using this apparatus the transmission of a $10 \mathrm{~m}$ PM fiber fitted with connectors was measured. The output polarizer was set to the principle axes of the fiber by optimizing the extinction. We refer to this as the $0^{\circ}$ position (referring to the angular rotation from alignment with the principle axes). In this first measurement the output polarizer was fixed and the angle of the input polarization was varied. The results of these measurements are shown in Fig. 6 (a). The periodic nature of the transmission is readily apparent and has FSR of $\sim 50 \mathrm{GHz}$, as expected for $10 \mathrm{~m}$ fiber of polarization dispersion $2 \mathrm{ps} / \mathrm{m}$. One sees that the AM measured for $45^{\circ}$ output polarization is $~ 12 \%$ peak-to-peak, and is reduced to less than about $1 \%$, and is in the experimental noise when aligned at $0^{\circ}$. With the output polarizer set at $45^{\circ}$ there is $1: 1$ mixing between the fast and slow polarization modes, and thus the AM is a measure of the mixing at the fiber input. In this case, following the analysis of Eq. (2), one expects the AM to be $4 \sqrt{P}$, where $P$ is the extinction. Thus for the measured extinction of this fiber of 1000:1, one calculates $\mathrm{AM}$ of $13 \%$, in agreement with the measurement. Based on this value our model predicts $\sim 0.4 \% \mathrm{AM}$ when the output polarizer is aligned at $0^{\circ}$.

To sufficiently eliminate this effect it would be required to attain extinction which is larger than available with current PM fibers. As a result we have investigated the use of "polarizing" or $\mathrm{PZ}$ fiber for this purpose. PZ fiber only allows one true propagating polarization mode. The orthogonal polarization is a leaky mode and has loss of $\sim 5 \mathrm{~dB} / \mathrm{m}$ of propagation. In Fig. 6 (b), PZ fiber is measured in the same arrangement used with the PM fiber (the output polarizer aligned at $\left.0^{\circ}\right)$. The data at both $0^{\circ}$ and $45^{\circ}$ input polarization show no significant AM. In addition, as a more stringent test, we have measured the transmission with both input and output misaligned by $45^{\circ}$ (curve shifted up in Fig. 6 (b)), and still no AM is apparent. 
"The issue of FM to AM conversion ...", J. E. Rothenberg et al
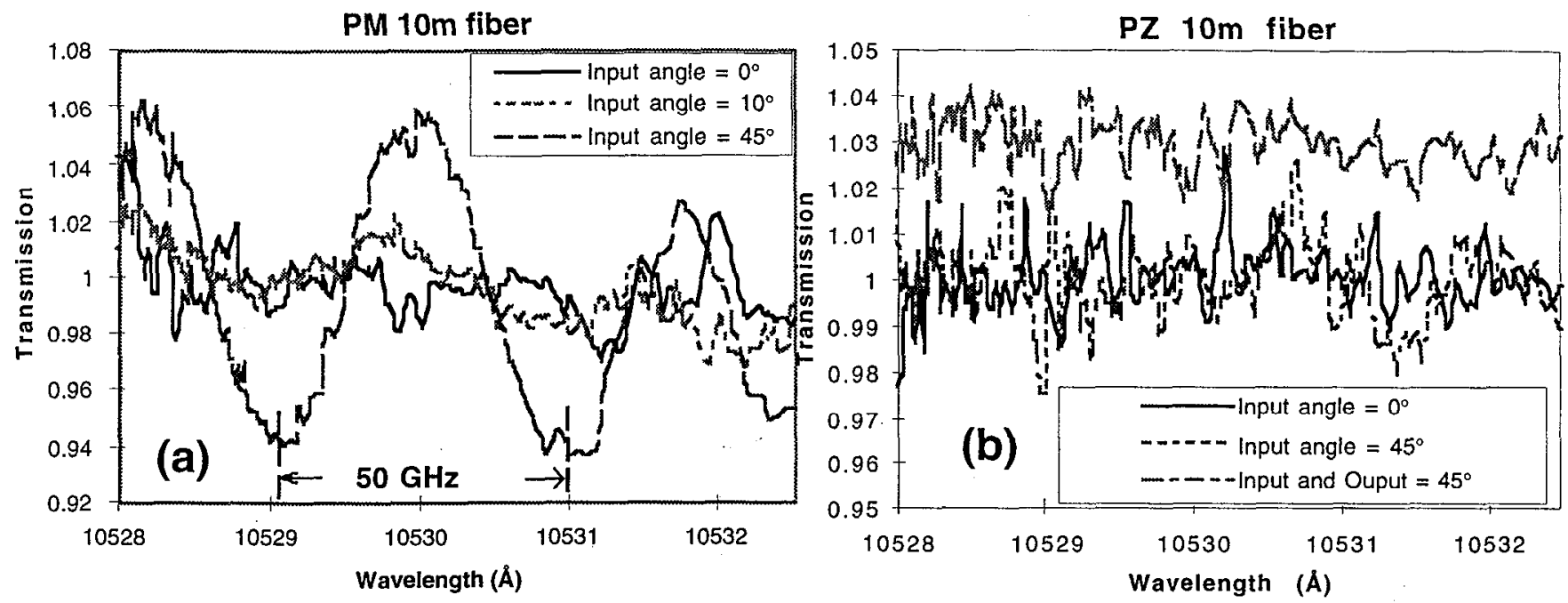

Figure 6: (a) Measured spectral transmission of $10 \mathrm{~m}$ PM fiber. Output polarizer has been aligned with the principle axes, and the input polarization angle is varied as indicated. (b) Transmission of a $10 \mathrm{~m} \mathrm{PZ}$ fiber measured in the same configuration. The measurement curve with both input and output polarization at $45^{\circ}$ has been shifted up for clarity.
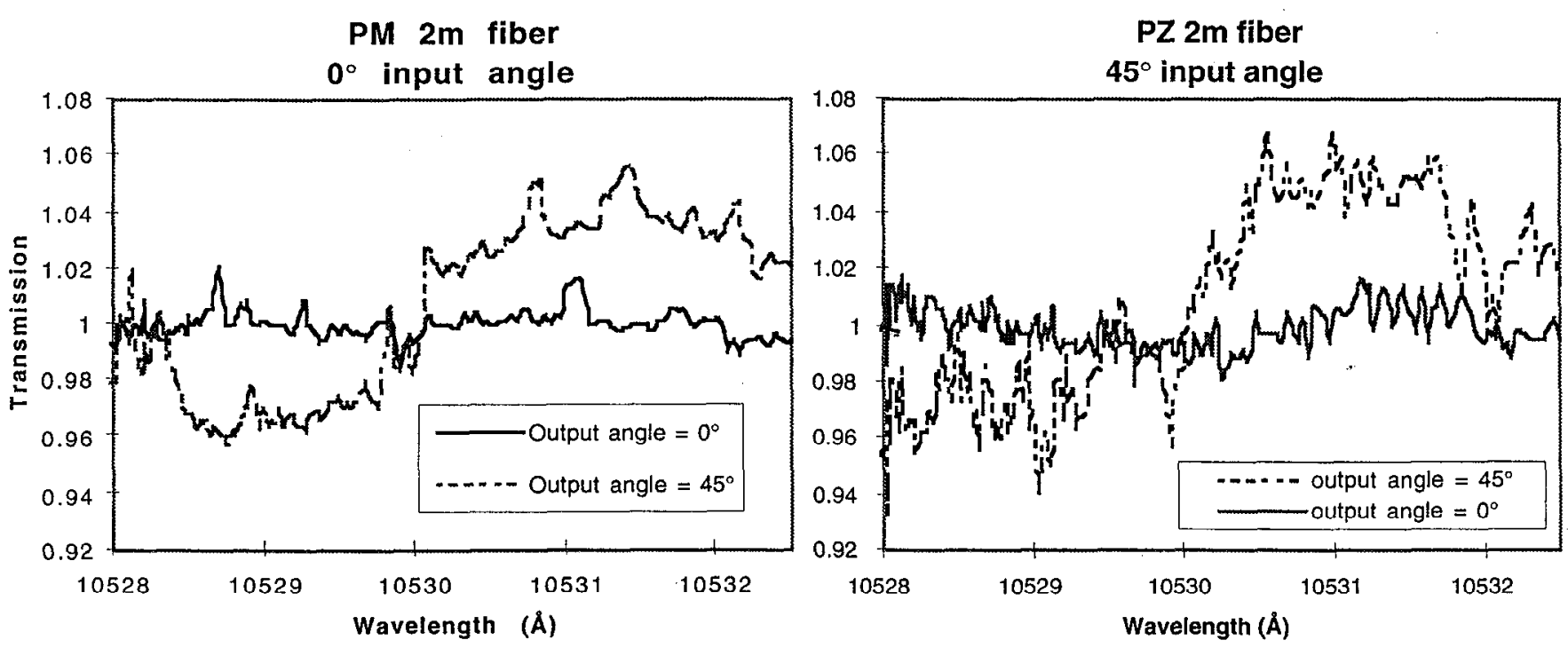

Figure 7: (a) Measured spectral transmission of $2 \mathrm{~m}$ PM fiber. Input polarizer has been aligned with the principle axes, and the output polarizer angle is varied as indicated. (b) Transmission of a $2 \mathrm{~m} \mathrm{PZ}$ fiber measured with $45^{\circ}$ input and $0^{\circ}$ output polarization (solid curve), and both input and output at $45^{\circ}$ (dashed curve).

These measurements were repeated with shorter $2 \mathrm{~m}$ fiber in Fig. 7. Again one observes significant AM $(\sim 10 \%)$ in the transmission of PM fiber with one polarizer aligned and the other at $45^{\circ}$. For $\mathrm{PZ}$ fiber in a comparable configuration to the PM fiber (i.e. input at $45^{\circ}$ and output 
at $0^{\circ}$ ) one observes no significant $\mathrm{AM}$ (solid curve). However, with both polarizers at $45^{\circ}$ one does observe some modulation. If one assumes a $20-30 \mathrm{~dB}$ cross-coupling between polarizations at the PZ fiber ends, one would then estimate that the AM in a $2 \mathrm{~m}$ PZ fiber is reduced below that found for PM fiber by a factor roughly equal to the square root of the cross coupling power, or a factor of 10-30. This result reflects the fact that after $2 \mathrm{~m}$ in PZ fiber the spurious polarization is still present in an appreciable, although significantly reduced amount.

$\triangle$ measurement of the effect of concatenation of two $10 \mathrm{~m}$ PM fibers is presented in Fig. 8. As with previous measurements, the AM is not significantly larger than the measurement noise when both polarizers are properly aligned. When the output polarizer is at $45^{\circ}$ one sees large AM of $\sim 20 \%$ in approximate agreement with expectations. The periodicity is now complicated by the coherent superposition of the interference effects in each fiber.

Two 10m PM fibers -concatenated

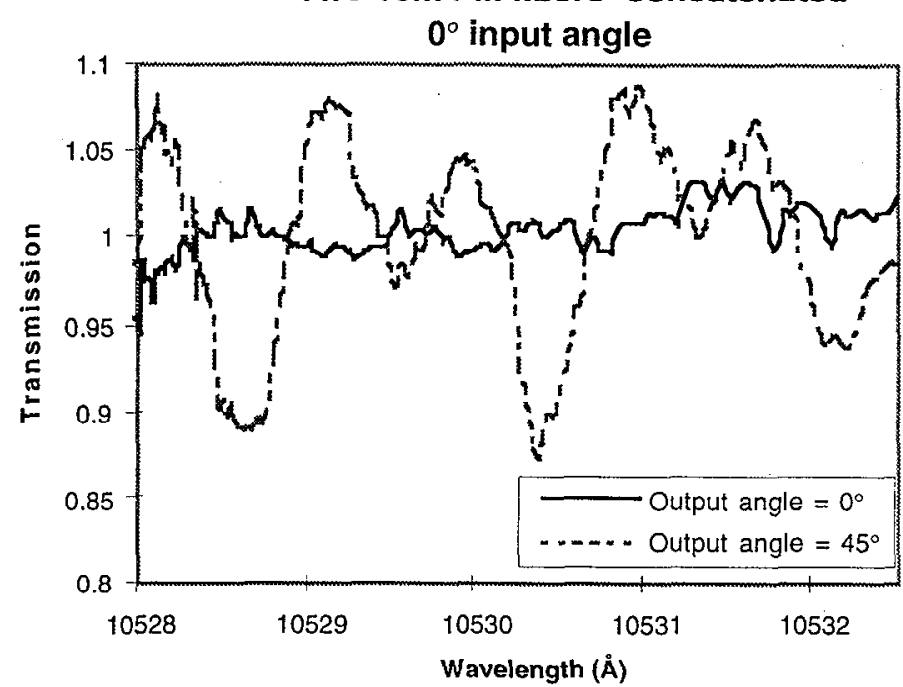

Figure 8: $\quad$ Measured spectral transmission of two $10 \mathrm{~m}$ PM fibers, joined with standard connectors. Input polarizer has been aligned with the principle axes, and the output polarizer angle is varied as indicated.

The measurements described above shows that the use of $\mathrm{PZ}$ fiber eliminates the AM originating from PM fiber components. However, there may be some components for which $\mathrm{PZ}$ is difficult to incorporate. One such kcy component in the fiber delivery system is the $1 \times 4$ fiber splitter. Because of the manufacturing process used for these devices it may be necessary to construct them with PM fiber. In Fig. 9 we measure the AM generated by a commercial (Canadian Instrumentation and Research) 1:4 splitter constructed with PM fiber. The polarization extinction of this splitter was measured at $22-30 \mathrm{~dB}$ for the four output ports. The length of fiber from input to the four output ports averaged $\sim 3.5 \mathrm{~m}$. In this measurement the input polarization is aligned $\left(0^{\circ}\right)$ to the principle axes. For each output port the polarizer angle is set at $45^{\circ}$. One sees that peak-to-peak AM is $\sim 10 \%$, which is similar to that obtained with a single fiber of $30 \mathrm{~dB}$ extinction. Given the poor polarization extinction of some ports one would expect larger levels of AM. However, the polarization coupling properties of the splitter have not been included in the simple model presented. If the output polarizer is aligned to $0^{\circ}$, the AM is again reduced to the level of our measurement noise. Thus, the limited use of a few PM fiber components should not lead to excessive amounts of AM. 
"The issue of FM to AM conversion ...", J. E. Rothenberg et al

However, these measurements do indicate that in order to keep AM at a modest level PZ fiber will be required for the majority of the components which transmit FM bandwidth.

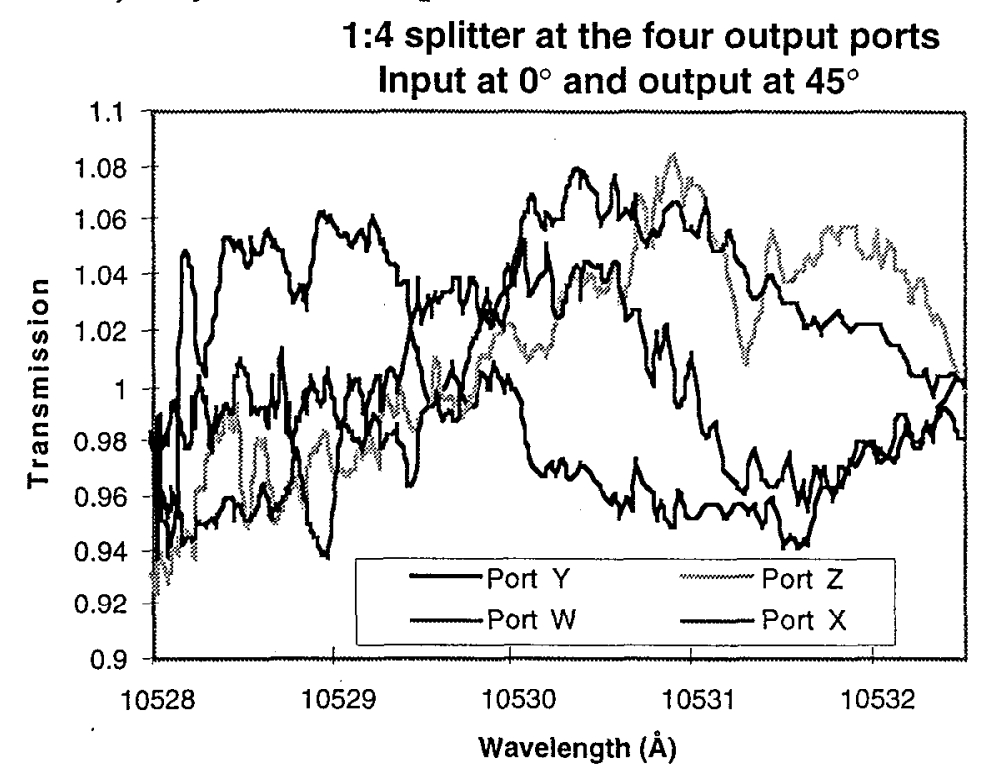

Figure 9: $\quad$ Measured spectral transmission of the four output ports of a 1:4 fiber splitter manufactured with PM fiber. Input polarizer has been aligned with the principle axes, and the output polarizer angle is set at $45^{\circ}$.

\section{AM FROM GAIN VARIATION OVER THE FM BANDWIDTH}

For frequencies very close to the gain peak one can expand the gain cross section as a Taylor series and keep only the first (quadratic) term. The gain can then be written

$$
G(\Delta \lambda)=\exp \left[g_{0}\left(1-b \cdot \Delta \lambda^{2}\right)\right]
$$

where $\Delta \lambda$ is the detuning from line center and $g_{0}$ is the line center gain. One finds the transmission relative to the peak gain on line center is then given by

$$
T(\Delta \lambda)=\exp \left[-g_{0} b \cdot \Delta \lambda^{2}\right]
$$

Thus, for small detuning from line center the exponential loss is simply quadratic in detuning, and we can write the loss in $\mathrm{dB}$ as

$$
T(\Delta \lambda)[\mathrm{db}]=-\mathrm{g}_{0}[\mathrm{db}] \cdot\left(\Delta \lambda / \lambda_{h w}\right)^{2}
$$

where $g_{0}$ is the line center gain in $\mathrm{dB}$ and represents the contribution of gain narrowing in this formula, and $\lambda_{h w}$ is a measure of the gain half-width in the absence of gain-narrowing. For example, one finds that for LG $760 \lambda_{h w} \sim 58 \AA$, and for LG $750 \lambda_{h w} \sim 80 \AA$. As an example consider a gain $g_{0}$ of $100 \mathrm{~dB}$ (typical of the NIF regenerative amplifier) and assume the gain medium is LG 760. Applying Eq. (10) one finds that the loss off line center is given by $T(\Delta \lambda)[\mathrm{db}]=-(\Delta \lambda / 5.8 \AA)^{2}$, and for small detuning this expression translates to a loss in percent of $(\Delta \lambda / 1.3 \AA)^{2}$. Thus, for $5 \AA \mathrm{FM}$ bandwidth centered on the gain line, the peak-topeak $\mathrm{AM}$ is $3.8 \%$. However, if one were to operate $5 \AA$ off line center with the same $5 \AA$ bandwidth the AM would increase to $\sim 30 \%$. Of course, since the gain variation around line 
"The issue of FM to AM conversion ...", J. E. Rothenberg et al

center can be precisely measured one could insert a spectral (loss) filter to compensate and flatten the transmission over the bandwidth of interest.

\section{CONCLUSIONS}

Conversion of FM to AM originates from a large number of physical effects found in a complex laser amplifier system. The fundamental cause of AM in all these effects is spectral variation in the transmission phase or amplitude of the system. The most severe AM has been found to originate in the fiber front end, where depolarization in polarization maintaining (PM) fiber components has been identified as the source, and the AM is found to increase linearly with the number of such components. The use of polarizing (PZ) fiber has been demonstrated to eliminate this source of AM. One must also take care to eliminate etalon effects throughout the laser system by either wedging optics or by AR coating. AM caused by group velocity dispersion can be eliminated by compensation with a dispersive delay line. Variation of gain over the laser bandwidth can be minimized by centering the FM bandwidth on the gain line center, however it would be possible to compensate for this variation with an appropriate spectral filter. The preferred diagnostic for determining the magnitude and source of FM to AM conversion is a precise spectral transmission scan over the bandwidth of interest.

\section{ACKNOWLEDGMENT}

This work was performed under the auspices of the U. S. Department of Energy by Lawrence Livermore National Laboratory under Contract No. W-7405-Eng-48.

\section{REFERENCES}

1. S. Skupsky, R. W. Short, T. Kessler, R. S. Craxton, S. Letzring, and J. M. Soures, "Improved laser-beam uniformity using the angular dispersion of frequency-modulated light," J. Appl. Phys. 66, 3456-3462 (1989). 